\title{
Rencontre avec Jean-Pierre Vincent, à propos de son Macbeth
}

\section{(2) OpenEdition \\ Journals}

Édition électronique

URL : http://journals.openedition.org/shakespeare/1318

DOI : $10.4000 /$ shakespeare.1318

ISSN : 2271-6424

Éditeur

Société Française Shakespeare

Édition imprimée

Date de publication : 1 novembre 1989

Pagination : 103-108

Référence électronique

"Rencontre avec Jean-Pierre Vincent, à propos de son Macbeth ", Actes des congrès de la Société française Shakespeare [En ligne], 7| 1989, mis en ligne le 01 janvier 2007, consulté le 05 mai 2019. URL : http://journals.openedition.org/shakespeare/1318; DOI : 10.4000/shakespeare.1318 


\title{
Société Française Shakespeare
}

\author{
Actes des Congrès \\ 1985 - 1986 - 1987
}

\section{LA FOLIE}

\section{SHAKESPEARE ET LES ARTS \\ LE TRAGIQUE}




\section{RENCONTRE AVEC J.P. VINCENT, à PROPOS DE SON MACBETH}

Dimanche 24 novembre 1985 , 14h30, ENS Ulm, Salle 46.

J.P. Vincent est présenté par J.M. DESPRATS.

Président de séance, Jean Fuzier

Secrétaire, André LASCOMBES

J.M. DESPRATS amorce le débat en demandant à J.P. Vincent s'il n'avait pas eu le projet initial de monter Le Roi Lear.

J.P. VINCENT voulait travailler une «grande» pièce afin de résoudre un maximum de difficultés. $K . L$. est la plus fréquemment montée, et par ailleurs, dans ses lectures Macbeth est revenue ; elle s'est imposée aussi par sa proximité à 1'actualité : la peur, qu'elle soit réelle ou fantasmée. Et encore à cause des parentés qu'elle entretient avec Woyseck d'une part (le fragmentaire, le trou), avec $K . L$. et Fin de Partie d'autre part. Enfin, l'intense brièveté des scènes, au cours desquelles les personnages changent, muent, est bien supérieure à ce qu'on trouve dans les autres pièces de Shakespeare, et en tout cas du répertoire français. Pièce atroce, insoutenable pour les acteurs.

J. FUZIER. - C'est une pièce rarement montée.

J.P. VINCENT - Pièce qui mérite sa réputation maudite : accidents d'acteurs, malaise réel aux répétitions. Rien à quoi s'accrocher dans la pièce ; déconcertante. Au terme du travail, pense que l'on peut la résumer ainsi : elle exprime tout ce que peut supporter un homme, et c'est énorme, trop ; toute une suite de pensées niées et d'échecs. L'histoire d'un homme ballotté entre des contradictions, un peu celle d'Adam/Eve au sortir de la grâce ; c'est le même abominable programme.

H. SUHAMY. - C'est une pièce facile à la lecture mais qui exigerait, à la réalisation, les ressources cinématographiques pour transcrire le tempo. De plus, les acteurs, qui cherchent toujours à composer un personnage, sont ici aux prises avec Adam, Everyman.

R. ELLRODT. - C'est pourtant une pièce structurellement facile parce que linéaire, clairement rythmée par les nombreuses références au jour et à la nuit. J.P. VINCENT - C'est vrai, si l'on en reste au niveau de l'analyse du 
texte. Mais un metteur en scène ne peut aujourd'hui en rester à la lecture universitaire. Il lui faut respecter ces virages à $180^{\circ}$ de l'âme de Macbeth, et qui font que dans la pièce le noir est clair et le clair est noir. D'autre part, c'est une pièce courtisane où s'exprime à la fois la flatterie au prince dans le thème du monde nouveau, et aussi un racisme anti-ecossais.

J.M. DESPRATS. - (en réponse à un étudiant) : Il n'y a pas de parti pris du noir ; c'est une pièce noire.

J.P.VINCENT. - Il y a bien un moment lumineux, lorsque M. devenu roi, laisse éclater un certain génie politique. Mais il apparaît vite inadapté aux choses du dessous. Il faut donc constamment jouer entre le noir et le clair. Monter Macbeth, voilà un mal qu'il ne souhaiterait pas à son pire ennemi. Macbeth ?

J.M. DESPRATS. - Pourquoi le choix de Philippe... pour jouer

J.P.VINCENT. - A cause de sa poésie innée, très personnelle. Encore qu'il ait fallu beaucoup s'opposer à lui lors du travail. Il faudrait en fait un acteurcaoutchouc pour jouer ce rôle; très peu en sont capables.

J. FUZIER. - Pourquoi a-t-il choisi le registre de la dérision?

J.P. VINCENT - Il est vrai que c'est un choix qui comporte et des gains et des pertes pour le rôle. (à R. ELLRODT) Non, il n'est pas mieux fait pour jouer Hamlet, mais il y a toujours ici le problème des changements de ton.

A. LASCOMBES. - La diction des acteurs, surtout celle de Philippe... entraînait des pertes considérables ; le texte paraissait trop confiné à la scène. Estce accident ou choix d'un style?

J.P.VINCENT. - C'est une pièce de cour faite pour la proximité ; absolument pas faite pour Avignon. Or, le Thêatre Français a depuis les travaux (remplacement du bois par le béton) la plus mauvaise acoustique de Paris. De plus l'acteur est fatigué.

D. GOY-BLANQUET. - Il y a quinze jours il semblait y avoir un partipris comique tout à fait délibéré.

J.P.VINCENT. - Oui, la scène du banquet par exemple peut être reçue sur le mode comique (la noce chez les petits bourgeois). Par ailleurs, l'acteur a une vision ironique et critique de ce qu'il joue ; il refuse l'emphase, par pudeur. Et la mise en scène ne cherche pas à dissimuler les contradicitons, elle ne «frime» pas.

(à SUHAMY qui remarque que l'angliciste français tient à retrouver la musique de Shakespeare) Le travail de DESPRATS conserve, dans la traduction, une musique comparable. Mais on ne peut pas sacrifier la violence des situations à la restitution lyrique. Or les acteurs français sont mal entrainés à conjuguer les deux registres : pour eux, c'est plus facilement ou Zola ou Racine.

(à FUZIER qui confirme que c'est une pièce intimiste, qui marche bien dans un cadre réduit). C'est à la fois une pièce «coup de poing» et une pièce fragile.

R. ELLRODT. - A aimé la sobriété de la mise en scène, et tout 
particulièrement ce cadre rectangulaire qui enchasse si heureusement l'apparition dorée du roi et de sa reine. Le lieu élisabéthain n'eût-il pourtant pas été plus efficace ? Pourquoi, par ailleurs, certaines coupures (la guérison des écrouelles), et à l'inverse avoir maintenu la scène des quatre sorcières?

J.P.VINCENT. - Des coupures à Paris, par souci de recentrer l'action sur les grands protagonistes, mais elles sont finalement moindres que celles pratiquées par les anglais.

Quant à la scène des quatre, elle est importante en ce que les sorcières n'y ont plus le même rôle. Elles sont servantes désormais. La scène, où la déesse tient le grand jeu, raconte la 2ème partie de la pièce, énonce le programme. Il était donc essentiel de la conserver.

J. PERRIN. - C'est une pièce confinée, plutôt qu'intimiste. Sous un ciel bas. Les acteurs éprouvent-ils cette sensation d'étouffement?

J.P.VINCENT. - C'est précisément ce que rend le prosaïsme de Thévenot dans la scène du banquet. C'est une pièce où le sentiment du poids varie constamment, mais est toujours là sauf au début du III. II faut encore souligner cette variabilité du personnage de Macbeth.

J.P. MOREAU. - Ne voit pas un état de grâce après le crime ; cette interprétation le gêne.

J.P.VINCENT. - La pièce est plutôt comme un cauchemar continuel où il faut ménager des moments de respiration. Macbeth est un homme qui fuit les problèmes, les remet à plus tard. styliste...)

(à J. M. DESPRATS qui demande pourquoi il a confié les costumes à un

J.P.VINCENT. - J'ai voulu à Paris une cohérence qui n'existait pas à Avignon. Le créateur choisi a donc retenu des costumes qui signent un passé historique par rapport à la date de la représentation : 1570 , soit 40 ans plus tôt. 